\title{
ELECTRONIC PROPERTIES OF SEMICONDUCTORS
}

\section{Study of the Effect of Doping on the Temperature Stability of the Optical Properties of Germanium Single Crystals}

\author{
O. I. Podkopaev ${ }^{a}$, A. F. Shimanskiy ${ }^{b *}$, S. A. Kopytkova ${ }^{a}$, R. A. Filatov ${ }^{a}$, and N. O. Golubovskaya ${ }^{b}$ \\ a Joint-Stock Company “Germanium”, Krasnoyarsk, 660027 Russia \\ ${ }^{b}$ Siberian Federal University, Krasnoyarsk, 660047 Russia \\ *e-mail: shimanaf@mail.ru
}

Submitted September 22, 2015; accepted for publication March 17, 2016

\begin{abstract}
The effect of doping on the optical transmittance of germanium single crystals is studied by infrared Fourier spectroscopy. It is established that the introduction of silicon and tellurium additives into germanium doped with antimony provides a means for improving the temperature stability of the optical properties of the crystals.
\end{abstract}

DOI: $10.1134 / \mathrm{S} 1063782616100213$

\section{INTRODUCTION}

The production of optical devices operating in the infrared (IR) spectral region is the widest field of application of single-crystal germanium. The main requirements to single-crystal germanium as an optical material are maximal transparency, a high degree of optical homogeneity, and the minimal number of defects. In the operating wavelength range from 2.5 to $11 \mu \mathrm{m}$, the room-temperature absorption coefficient should be about $0.02 \mathrm{~cm}^{-1}[1,2]$. In Ge, absorption at free charge carriers is dominant. The specific feature of such a mechanism of absorption is that the photonabsorption cross section of holes is about an order of magnitude larger than the corresponding parameter for electrons [3, 4]. For this reason, in order to exclude the effect of holes produced by background acceptor impurities, germanium is doped with donor additives, commonly with antimony. Antimony-doped germanium, whose resistivity ranges from 3 to $40 \Omega \mathrm{cm}$, is extensively used in the production of optical elements, such as lenses, windows, filters, etc. In the absence of small-angle boundaries, such material purified to the proper degree and containing less than $10^{4} \mathrm{~cm}^{-2}$ of dislocations exhibits high transparency, about $46.0 \%$; the absorption coefficient $\alpha$ near the absorption band edge, at a wavelength of $10.6 \mu \mathrm{m}$ is $0.015-0.035 \mathrm{~cm}^{-1}$ $[1,5,6]$. An important disadvantage of optical germanium is substantially reduced transparency upon heating to temperatures above $45^{\circ} \mathrm{C}$. For example, at $60^{\circ} \mathrm{C}$, the absorption coefficient of the crystals, whose resistivity is $3-5 \Omega \mathrm{cm}$, is increased to $\sim 0.065 \mathrm{~cm}^{-1}[7,8]$. At higher resistivities, the absorption coefficient is even higher.
One of the methods of improving the temperature stability of the optical properties of Ge-based IR optical devices is the use of chalcogenide glasses of the type $\mathrm{Ge}-\mathrm{As}-\mathrm{Se}$, $\mathrm{Ge}-\mathrm{As}-\mathrm{Te}$, etc. [9, 10].

Another approach is based on the doping of germanium with various additives. However, this approach has not been adequately developed so far, and few data on the effect of doping on the temperature behavior of the optical properties are of qualitative character [11, 12].

In this context, the purpose of this study is to explore the effects of silicon and tellurium additives on the temperature stability of the optical transmittance of germanium single crystals doped with antimony.

The choice of silicon and tellurium as dopant additives is governed by a number of considerations. At temperatures higher than the donor exhaustion temperature, the maximum concentration of electrons appearing in germanium as a result of the ionization of antimony is equal to the concentration of donor atoms: $n_{\mathrm{d}}=N_{\mathrm{d}}$. The concentration of thermally generated intrinsic holes is defined by the relation

$$
p_{\mathrm{i}}=N_{\mathrm{V}} \exp \left(-E_{\mathrm{g}} / 2 k_{\mathrm{B}} T\right),
$$

where $E_{\mathrm{g}}$ is the band gap and $N_{\mathrm{v}}$ is the density of electron states in the valence band. It is obvious that the critical temperature $T_{\mathrm{cr}}$, starting from which intrinsic charge carriers are dominant in the semiconductor and the influence of the donor additive is limited, corresponds to the condition $n_{\mathrm{d}}=p_{\mathrm{i}}$ or

$$
n_{\mathrm{d}}=N_{\mathrm{d}}=p_{\mathrm{i}}=N_{\mathrm{v}} \exp \left(-E_{\mathrm{g}} / 2 k_{\mathrm{B}} T_{\mathrm{cr}}\right) \text {. }
$$




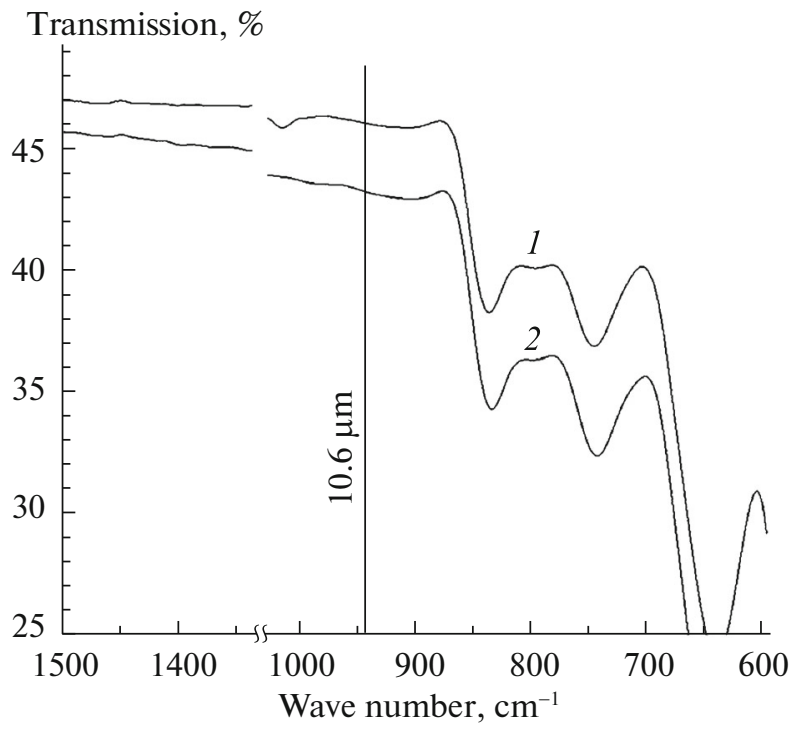

Fig. 1. IR transmittance spectra of the Sb-doped Ge crystal with a resistivity of $3 \Omega \mathrm{cm}$ at a temperature of $T=(1)$ 20 and $(2) 60^{\circ} \mathrm{C}$.

Hence,

$$
T_{\text {cr }}=\frac{E_{\mathrm{g}}}{\ln \left(N_{\mathrm{v}} / N_{\mathrm{d}}\right) 2 k_{\mathrm{B}}} .
$$

Consequently, to increase the temperature $T_{\mathrm{cr}}$ and, thus, to improve the temperature stability of the semiconductor properties, it is necessary either to increase the concentration of the donor impurity or to attain an increase in the band gap $E_{\mathrm{g}}$. For germanium to be used in the optical industry, the first-mentioned method is inefficient, since an increase in the concentration of antimony yields a significant increase in absorption due to impurity disordering of the germanium crystal lattice [5]. Thus, the most appropriate method is the introduction of silicon into germanium.

A similar approach was used in [12]. To improve the thermal stability of the electrical properties of Sbdoped Ge crystals, Krishtopenko et al. [12] introduced equal amounts ofelectrically neutral $\mathrm{Pb}$ and $\mathrm{Si}$ impurities in addition to the $\mathrm{Sb}$ impurity. However, in [12], the effect of doping on the optical characteristics of the crystals was not considered. Tellurium was chosen for the dopant additive, since in germanium, tellurium can be doubly ionized and create deep levels at 0.1 and $0.3 \mathrm{eV}$, which can influence the optical properties of crystals at high temperatures.

\section{EXPERIMENTAL}

Germanium single crystals were grown in a quartz crucible by the Czochralski method. Growth was conducted using a Redmet-type system, in an argon atmosphere at an excess pressure of $0.02 \mathrm{MPa}$. The mass of the zone-purified polycrystalline germanium charge was $4 \mathrm{~kg}$. The diameter of the ingots grown was $30-40 \mathrm{~mm}$. The growth direction corresponded to the [111] crystallographic direction. The rate of rotation of the crucible was $6 \mathrm{rpm}$, the rate of rotation of the seed was $20 \mathrm{rpm}$, and the lifting speed of the seed was $0.5 \mathrm{~mm} \mathrm{~min}^{-1}$. Sb- and Te-dopant additives were used in the form of $\mathrm{Ge}-\mathrm{Sb}$ and $\mathrm{Ge}-\mathrm{Te}$ alloys. The required amount of $\mathrm{Si}$ was added as a pure element.

The crystals grown were used for the preparation of polished samples shaped as 1-cm-thick plane-parallel wafers to be used for determining the optical characteristics. The optical transmittance spectrum was recorded in the wavelength range from 2.5 to $16.6 \mu \mathrm{m}$ (or at wave numbers in the range from 4000 to $600 \mathrm{~cm}^{-1}$ ) using a SPECTRUM BXII IR Fourier spectrometer. The accuracy in determining the optical transmittance was $\pm 0.1 \%$. To carry out measurements with increasing temperature to $60^{\circ} \mathrm{C}$, we used a heating attachment that provided stable thermostating of the sample with an accuracy of $\pm 0.1^{\circ}$.

From the spectra, we determined the optical transmittance $T$ at a wavelength of $10.6 \mu \mathrm{m}$ and calculated the absorption coefficient by the formula

$$
\alpha=-\frac{1}{t} \ln \left(\frac{(1-r)^{4}}{4 r^{4} T^{2}}+\frac{1}{r^{2}}\right)-\frac{(1-r)^{2}}{2 r^{2} T},
$$

Here, $t$ is the sample thickness (in $\mathrm{cm}$ ), $\alpha$ is the absorption coefficient, and $r$ is the reflectance.

\section{RESULTS AND DISCUSSION}

Figure 1 shows the IR transmittance spectra in the range of wave numbers from 600 to $1500 \mathrm{~cm}^{-1}$ for the $\mathrm{Sb}$-doped Ge crystal with a resistivity of $3 \Omega \mathrm{cm}$ at room temperature and $60^{\circ} \mathrm{C}$.

From the data shown in Fig. 1, it follows that, for the sample at room temperature, the optical transmittance of IR radiation at a wave number of $943 \mathrm{~cm}^{-1}$ corresponding to the wavelength $10.6 \mu \mathrm{m}$ is $46.0 \%$. An increase in the temperature yields a decrease in the transmittance. At a temperature of $60^{\circ} \mathrm{C}$, the optical transmittance at $10.6 \mu \mathrm{m}$ is $43.10 \%$.

As the resistivity of the sample is increased to $7 \Omega \mathrm{cm}$ because of a decrease in the content of antimony in the crystal, the transmittance at room temperature remains practically unchanged. At the same time, as temperature is elevated, the transmittance decreases much more noticeably compared to the transmittance of the sample with the resistivity $3 \Omega \mathrm{cm}$ and reaches $42.80 \%$, which corresponds to a lower concentration of donor electrons involved in the compensation of thermally activated intrinsic holes. The results of experiments performed in this study correlate with well-known data reported in [8]. According to the data of [8], the highest degree of thermal stability is exhibited by Ge crystals with a resistivity of $3 \Omega \mathrm{cm}$ corre- 
Optical parameters of germanium single crystals at the wavelength $10.6 \mu \mathrm{m}$ in relation to the concentration of dopants in the melt and to temperature

\begin{tabular}{c|c|c|c|c|c|c|c|c}
\hline \multirow{2}{*}{ No. } & \multicolumn{2}{|c|}{$\begin{array}{c}\text { Concentration of dopant additives } \\
\text { in the melt, } \mathrm{cm}^{-3}\end{array}$} & \multirow{2}{*}{$\begin{array}{c}\text { Resistivity at } \\
T=20^{\circ} \mathrm{C}, \Omega \mathrm{cm}\end{array}$} & $\begin{array}{c}\text { Optical } \\
\text { transmittance, } \\
\%\end{array}$ & $\begin{array}{c}\text { Absorption } \\
\text { coefficient, } \\
\mathrm{cm}^{-1}\end{array}$ & $\begin{array}{c}\text { Optical } \\
\text { transmittance, } \\
\%\end{array}$ & $\begin{array}{c}\text { Absorption } \\
\text { coefficient, } \\
\mathrm{cm}^{-1}\end{array}$ \\
\cline { 2 - 4 } & $\mathrm{SB}$ & $\mathrm{SI}$ & $\mathrm{TE}$ & & \multicolumn{2}{|c|}{ Temperature $20^{\circ} \mathrm{C}$} & \multicolumn{2}{|c|}{ Temperature $60^{\circ} \mathrm{C}$} \\
\hline 1 & $2.5 \times 10^{17}$ & - & - & $\sim 3.0$ & 46.00 & 0.0168 & 43.10 & 0.0652 \\
2 & $2.5 \times 10^{17}$ & $0.5 \times 10^{20}$ & - & $\sim 3.0$ & 46.00 & 0.0168 & 43.20 & 0.0634 \\
3 & $2.5 \times 10^{17}$ & $1.2 \times 10^{20}$ & - & $\sim 3.0$ & 46.00 & 0.0168 & 43.30 & 0.0615 \\
4 & $2.5 \times 10^{17}$ & $0.5 \times 10^{20}$ & $5.0 \times 10^{19}$ & $\sim 3.0$ & 46.20 & 0.0134 & 43.40 & 0.0597 \\
5 & $2.5 \times 10^{17}$ & $1.2 \times 10^{20}$ & $5.0 \times 10^{19}$ & $\sim 3.0$ & 46.20 & 0.0134 & 43.50 & 0.0578 \\
6 & $3.0 \times 10^{16}$ & - & - & $\sim 7.0$ & 45.90 & 0.0185 & 42.80 & 0.0699 \\
7 & $3.0 \times 10^{16}$ & $1.2 \times 10^{20}$ & - & $\sim 7.0$ & 46.00 & 0.0168 & 42.90 & 0.0680 \\
8 & $3.0 \times 10^{16}$ & $1.2 \times 10^{20}$ & $5.0 \times 10^{19}$ & $\sim 7.0$ & 46.10 & 0.0151 & 43.00 & 0.0670 \\
\hline
\end{tabular}

sponding to an antimony concentration in the crystal of $7 \times 10^{14} \mathrm{~cm}^{-3}$.

From the optical transmittance, we calculated the IR absorption coefficients for crystals with resistivities of 3 and $7 \Omega \mathrm{cm}$ and found that, at room temperature, the absorption coefficients were 0.0168 and $0.0185 \mathrm{~cm}^{-1}$, respectively (see table).

In order to improve the thermal stability of the crystals, we introduced silicon and tellurium into the germanium melt simultaneously with the introduction of antimony. The amounts of silicon and tellurium corresponded to $(0.5-1.2) \times 10^{20} \mathrm{~cm}^{-3}$ and $(1-5) \times$

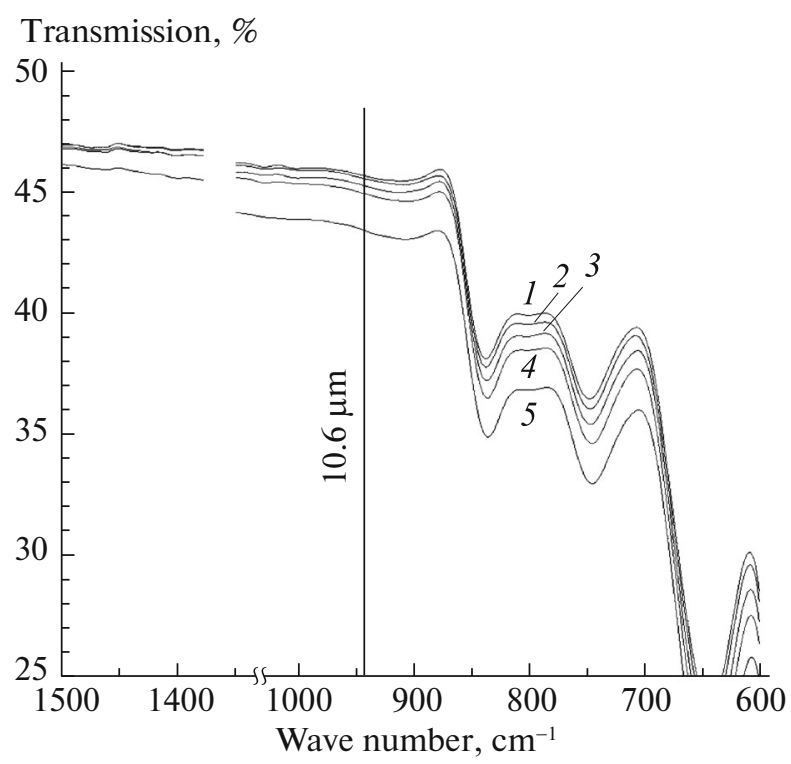

Fig. 2. IR transmittance spectra of the Sb-, Si-, and Tedoped Ge crystal with a resistivity of $3 \Omega \mathrm{cm}$ at a temperature of $T=$ (1) 20, (2) 30 , (3) 40, (4) 50 , and (5) $60^{\circ} \mathrm{C}$.
$10^{19} \mathrm{~cm}^{-3}$. According to the values of the effective distribution coefficient known from [13, 14], these amounts provided $\mathrm{Si}$ - and $\mathrm{Te}$-impurity concentrations in the crystal at a level of $(3.0-7.2) \times 10^{20} \mathrm{~cm}^{-3}(0.05-$ 0.15 at $\%)$ and $(1-5) \times 10^{13} \mathrm{~cm}^{-3}$, respectively. The limiting Si and Te concentrations are defined by the fact that, at lower concentrations, a positive effect is lacking. At concentrations higher than the above indicated limiting values, the structural quality of the crystals deteriorates.

The characteristics of experimental samples and the values of the optical transmittance and absorption coefficient at a wavelength of $\lambda=10.6 \mu \mathrm{m}$ at room temperature and the higher temperature are given in the table and Fig. 2.

It is established that, upon the introduction of the $\mathrm{Si}$ impurity into the $\mathrm{Sb}$-containing melt to a concentration in the range from $0.5 \times 10^{20}$ to $1.2 \times 10^{20} \mathrm{~cm}^{-3}$, the optical transmittance at the temperature $60^{\circ} \mathrm{C}$ increases to $43.30 \%$. In this case, the corresponding absorption coefficient is $0.0615 \mathrm{~cm}^{-1}$. In the case of triple doping, i.e., upon the introduction of tellurium along with antimony and silicon, the transmittance increases even more, to $43.50 \%$ at a concentration of tellurium in the melt of $5 \times 10^{19} \mathrm{~cm}^{-3}$. In this case, the absorption coefficient is $0.0578 \mathrm{~cm}^{-1}$.

\section{CONCLUSIONS}

The simultaneous introduction of antimony, silicon, and tellurium into the germanium melt provides a means for improving the temperature stability of the optical properties of the crystals grown and for attaining an IR absorption coefficient below $0.06 \mathrm{~cm}^{-1}$ at $60^{\circ} \mathrm{C}$. 


\section{REFERENCES}

1. L. Cor Claeys and E. Simoen, Germanium-Based Technologies: From Materials to Devices (Elsevier, Oxford, 2007) p. 18.

2. B. Depuydt, A. Theuwis, and I. Romandic, Mater. Sci. Semicond. Process. 9, 437 (2006).

3. R. H. Bube, Photoconductivity of Solids (Inostr. lit., Moscow, 1962; Krieger, New York, 1978), p. 259.

4. W. Kaiser, R. J. Collins, and H. Y. Fan, Phys. Rev. 91, 1380 (1954).

5. I. M. Nesmelova and N. I. Astaf'ev, Prikl. Fiz. 5, 33 (2007).

6. E. D. Capron and O. L. Brill, Appl. Opt. 12, 569 (1973).

7. K. A. Osmer, C. J. Pruszynski, and J. Richter, Proc. SPIE 1112, 83 (1989).

8. Umicore Germanium Optics Leading the Way in Infrared Optics. Data Analysis Software. http://eom.umi-
core.com/en/materials/library/brochuresAndMarketingMaterial/show_GermaniumOpticsBrochure.pdf (Cited April 29, 2015).

9. C. Marta de la Fuente, Proc. SPIE 6342, 63421C-1 (2007).

10. Y. Guimond, J. Franks, and Y. Bellec, Proc. SPIE 5406, 114 (2004).

11. I. Yonenaga, J. Cryst. Growth 226, 47 (2001).

12. N. V. Postikova and A. Ya. Gubenko, RF Patent No. 1461046 (1996).

13. I. Yonenaga, A. Matsu, S. Tozawa, and K. Sumino, J. Cryst. Growth 154, 275 (1995).

14. A. Ya. Nashel'skii, Production of Semiconductor Materials (Metallurgiya, Moscow, 1989), p. 55 [in Russian].

Translated by E. Smorgonskaya 